Remarquons que le théorème peut être faux sans l'hypothèse que $G$ soit connexe. En effet, soit $G=\{0,1\}$ un groupe de deux éléments, chacun de mesure $1 / 2$. Soit $A=B=\{O\}$. Alors, $A+B=\{O\}$.

THÉoRł̀me 2. Soit $G$ un groupe topologique compact quelconque. Soient $A, B C G$ mesurables avec $m(A)+m(B)>1$. Alors on a $A+B=G$.

En effet, soit $x \in G$. Alors, $m(x-A)=m(A)$, donc $(x-A) \cap B \neq \varphi$ (l'ensemble vide), ce qui implique que $x \in A+B$, c. q. f. d.

\section{Travaux cités}

[1] B. Eckmann, Über monothetische Gruppen, Com. Math. Helv. 16 (1943), p. $249-263$.

[2] P. R. Halmos and H. Sammelson, On monothetic groups, Proc. Nat. Acad. Sc. 28 (1942), p. 254-258

[3] A. M. Macbeath, On measure of sum sets II, Proc. Cambr. Philos. Soc. 49 (1953), p. 40-43.

[4] H. Ostmann, Verfeinerte Lösung der asymptotischen Dichtenaufgabe, Journ. Reine u. Ang. Math. 87 (1950), p. 183-188.

[5] Д. А. Р айков, О сложении жножеств в слысле Іенирельнана, Мат. Сборн. 5 (1939), p. 425-438.

Regu par la Rédaction le 31.3. 1954

\section{A property of plane homeomorphisms}

\section{by}

\section{H. G. Eggleston (Cambridge)}

In a real Euclidean plane $E$ let $(x, y)$ denote the Cartesian coordinates of a point $p$, and let $\Theta$ denote the set of all homeomorphisms of $E$ onto itself which are of the form

$$
(x, y) \rightarrow\left(x^{\prime}, y^{\prime}\right)
$$

where

$$
x^{\prime}=x, \quad y^{\prime}=\Phi(x, y) \quad \text { or } \quad x^{\prime}=\Phi(x, y), \quad y^{\prime}=y .
$$

It is supposed that either the first alternative holds for every point $(x, y)$ of $E$ or the second alternative holds for every point of $E$. Denote by $E$ the group formed by all finite superpositions of any of the transformations of $\Theta$. S. Ulam $^{1}$ ) has raised the question as to whether it is possible to approximate to any arbitrary homeomorphism of the plane onto itself by members of $\Xi$.

The solution of the problem depends upon the meaning to be assigned to the word "approximate". In $\S 1$ of this paper it is shown that if the approximation is to be uniform then the answer is in the negative, that is to say, if for any two homeomorphisms $\mathfrak{H}_{1}, \mathfrak{H}_{2}$ of the plane $E$ we write

$$
\delta\left(\mathfrak{S}_{1}, \mathfrak{S}_{2}\right)=\underset{p \in E}{\operatorname{up} . \mathrm{bd}} \varrho\left(\mathfrak{H}_{1}(p), H_{2}(p)\right)
$$

where $\varrho$ denotes the Euclidean distance, then a homeomorphism (5 can be constructed such that for any member $\mathfrak{S}$ of $\Xi, \delta(\mathfrak{H}, \mathfrak{G})>1$.

The example that is constructed here, depends essentially upon the fact that the plane is not compact. If we restrict ourselves to compact subsets the situation is different. In $\S \S 2$ and 3 we prove that if $S$ is a closed square with its sides parallel to the coordinate axes and if $\Theta^{\prime}$ is the subclass of the members of $\Theta$ which leave each frontier point of $S$ fixed and it $\Xi^{\prime}$ is the group generated by finite superpositions of mem- 
bers of $\Theta^{\prime}$; then given any plane homeomorphism $(5$ of $S$ onto itself that leaves the frontier points of $S$ fixed, there exists a sequence of homeomorphisms $\mathfrak{S}_{n}$ belonging to $\Xi^{\prime}$ such that

$$
\delta^{\prime}\left(\mathfrak{S}_{n}, \mathfrak{G}\right)=\operatorname{mp}_{p \in S} \text { bd. } \varrho\left(\mathfrak{H}_{n}(p), \mathfrak{G}(p)\right) \rightarrow 0 \quad \text { as } \quad n \rightarrow \infty .
$$

The result proved in $\S 3$ is used in $\S 4$ to show that if we interpret $\mathfrak{S}_{n} \rightarrow \mathfrak{G}$ as $n \rightarrow \infty$ to mean that $\mathfrak{H}_{n}(p) \rightarrow \mathfrak{G}(p)$ for every point $p$ of $E$ then to any given homeomorphism $(\mathfrak{G}$ corresponds a sequence of homeomorphisms $\mathfrak{H}_{n}$, belonging to $\Xi$ such that $\mathfrak{H}_{n} \rightarrow \mathfrak{G}$ as $n \rightarrow \infty$.

\section{\$ 1. Uniform approximation}

Let $A$ denote a simple arc with end-points $a_{1}, a_{2}$ in the plane $E$ and let $p$ be any point of the plane which does not belong to $A$. Let $q$ denote a variable point on $A$. Consider the change in direction of the line $p q$, directed from $p$ to $q$ when $q$ describes $A$ from $a_{1}$ to $a_{2}$. If we fix on a direction through $p$ from which to measure angles, say $p l$, take a particular sense of rotation as positive and assign a particular appropriate value to $\Varangle a_{1} p l$, then there will be two points (or more) of $A$ say $q^{\prime}$ and $q^{\prime \prime}$ such that for all $q$ of $A$,

Write

$$
\Varangle q^{\prime} p l \leqslant \Varangle q p l \leqslant \Varangle q^{\prime \prime} p l .
$$

$$
\begin{aligned}
& \Varangle q^{\prime \prime} p l-\Varangle q^{\prime} p l=\alpha(A, p), \\
& \beta(A)=\operatorname{up}_{p \in E-A} \text {. bd. } \alpha(A, p) .
\end{aligned}
$$

$\alpha(A, p)$ is independent of the sense of description of $A$ and of the particular way in which the angles $\Varangle q p l$ are measured. It is always a positive or zero number. It is also finite (for if it were infinite $A$ would wind round $p$ infinitely often and since $p$ is at a positive distance from $A$ this would mean that $A$ was not locally connected) but $\beta(A)$ may be infinite. However we shall use this function of $A$ only when it is finite. We then have the following lemma: then

LEMMA 1. If $\beta(A)$ is finite and $H$ is a homeomorphism of the class $\Theta$

$$
\beta(\mathfrak{S}(A)) \leqslant 2 \pi+\beta(A) .
$$

Suppose that $\mathfrak{H}$ is $(x, y) \rightarrow\left(x^{\prime}, y^{\prime}\right)$ where

$$
x^{\prime}=x, \quad y^{\prime}=\Phi(x, y)
$$

If $H$ is of the alternative form the argument is similar with $x$ and $y$ interchanged. Since when $p$ varies over $E-A, \mathfrak{S}(p)$ varies over $E-\mathfrak{H}(A)$ it is sufficient to show that for any $p$ of $E-A$

$$
\alpha(\mathfrak{H}(A), \mathfrak{H}(p)) \leqslant 2 \pi+\alpha(A, p) .
$$

In proving (4) we assume (without loss of generality since $a(A, p)$ varies continuously with $A$ ) that $A$ is a polygonal arc with no segments parallel to the $y$-axis. Let $A^{\prime}$ be a minimal subare of $A$ for which

$$
\alpha\left(\mathfrak{H}\left(A^{\prime}\right), \mathfrak{H}(p)\right)=\alpha(\mathfrak{H}(A), \mathfrak{S}(p)) .
$$

If $A^{\prime}$ degenerates to a single point the result is trivially true, otherwise let $p$ be the point $\left(x_{1}, y_{1}\right)$.

Firstly consider the case when $A^{\prime}$ does not intersect the line $x=x_{1}$. Then $\mathfrak{H}\left(A^{\prime}\right)$ does not intersect the line $x=x_{1}$. Thus, since this line contains the point $\mathfrak{S}(p)$,

$$
\alpha(\mathfrak{S}(A), \mathfrak{H}(p))=\alpha\left(\mathfrak{D}\left(A^{\prime}\right), \mathfrak{T}(p)\right) \leqslant \pi \leqslant 2 \pi+\alpha(A, p) .
$$

Next consider the case when $A^{\prime}$ does intersect the line $x=x_{1}$. Let $A^{\prime}$ have end-points $e, f$ and meet the line $x=x_{1}$ in points whose order on $A^{\prime}$ from $e$ to $f$ is $p_{1}, p_{2}, \ldots, p_{n}$ where $e$ may be $p_{1}$ and $f$ may be $p_{n}$. Since the subarc $e p_{1}$ of $A^{\prime}$ lies entirely (except for the point $p_{1}$ ) on one side of $x=x_{1}$, so does the subarc $\mathfrak{H}\left(e p_{1}\right)$ of $\mathfrak{H}\left(A^{\prime}\right)$. Similarly $\mathfrak{H}\left(f p_{2}\right)$ lies entirely on one side of $x=x_{1}$. Thus if the line $\mathfrak{H}(p) m$ is in a fixed direction through $\mathfrak{H}(p)$ we have,

Further

$$
\begin{array}{lc}
\text { (8) } & \left|\Varangle p_{n} p l-\Varangle p_{1} p l\right|=\left|\Varangle \mathfrak{H}\left(p_{n}\right) \mathfrak{S}(p) m-\Varangle \mathfrak{S}\left(p_{1}\right) \mathfrak{S}(p) m\right| \\
\text { and } & \alpha(A, p) \geqslant \alpha\left(A^{\prime}, p\right) \geqslant\left|\Varangle p_{n} p l-\Varangle p_{1} p l\right| .
\end{array}
$$

Thus from $(5),(\bar{T}),(8),(9)$

$$
\alpha(\mathfrak{H}(A), \mathfrak{H}(p)) \leqslant 2 \pi+\alpha(A, p),
$$

and this is the required inequality (4).

We can now prove the main result which we state as a theorem. THEOREM 1. There are homeomorphisms of the plane onto itself which are not the uniform limit of any sequence of members of $\boldsymbol{\Xi}$.

Let $K_{n}$ be the are whose equation in polar coordinates is

$$
\text { (11) } r=\left(4+e^{-\theta}-5 e^{-2 n \pi}\right)\left(1-e^{-2 n \pi}\right)^{-1}, \quad 0 \leqslant \theta \leqslant 2 \pi ; \quad n=1,2, \ldots
$$


This are is part of a spiral which starts at $r=5, \theta=0$ winds round the origin $n$ times with $r$ monotonically decreasing and ends at the point $r=4, \theta=2 n \pi$.

Denote by $M_{n}$ the are obtained from $K_{n}$ by a translation parallel to the $x$-axis by an amount $10 n$. The arcs $M_{1}, M_{2}, \ldots, M_{n}, \ldots$ are disjoint. There is a homeomorphism of the plane onto itself which maps the segment $L_{n}=\{(x, y) ; 10 n-1 \leqslant x \leqslant 10 n, y=0\}$ onto the are $M_{n}$, $n=1,2, \ldots$ Denote this homeomorphism by $\mathfrak{G}$ and let $\mathfrak{H}$ be a member of $\Xi$ which we may suppose is obtained by the superposition of the members $\mathfrak{H}_{1}, \mathfrak{S}_{2}, \ldots, \mathfrak{H}_{s}$ of $\Theta$.

Then $\beta\left(L_{n}\right)=\pi$ and thus by lemma 1 ,

$$
\beta\left(\mathfrak{H}\left(L_{n}\right)\right) \leqslant(2 s+1) \pi, \quad n=1,2, \ldots
$$

Consider the segment $L_{s+1}$. Let $q$ be the point with coordinates $x=10(s+1), y=0$. Write $p=\mathfrak{5}^{-1}(q)$. Then

$$
\alpha\left(\mathfrak{b}\left(L_{s+1}\right) \mathfrak{G}(p)\right)=\alpha\left(M_{s+1}, q\right)=(2 s+2) \pi .
$$

Now if it were the case that $\delta\left(\mathfrak{S},(\mathfrak{b}) \leqslant 1\right.$, then $\mathfrak{H}\left(L_{s+1}\right)$ would be an are whose end-points would be distant at most 1 from the end-points of $\mathfrak{5}\left(L_{s+1}\right)=M_{s+1}$ and which is such that when $p^{\prime}$ describes $L_{s+1}$ the points $\mathfrak{S}\left(p^{\prime}\right)$ and $\mathfrak{G}\left(p^{\prime}\right)$ are distant apart at most 1 . But under these circumstances $\mathfrak{5}\left(p^{\prime}\right)$ winds round the point $q s+1$ times keeping at a distance of at least 4 from it. Thus $\mathfrak{S}\left(p^{\prime}\right)$ also winds round $q s+1$ times except that its end-points may be such that it fails to complete the $(s+1)$ th rotation by an angle less than $\left.2 \sin ^{-1}(1 / 4)^{2}\right)$. Hence

$$
\alpha\left(\mathfrak{S}\left(L_{s+1}\right), \mathfrak{5}(p)\right) \geqslant(2 s+2) \pi-2 \sin ^{-1}(1 / 4)>(2 s+1) \pi .
$$

Since the inequalities (12) and (14) are in contradiction with one another, $\delta(\mathfrak{H},(\mathfrak{b})>1$ and the theorem is proved.

\section{2. Homeomorphisms of certain subsets of a closed square \\ onto themselves}

Let $S$ be a closed square with sides parallel to the coordinate axes. We shall consider approximations to homeomorphisms of $S$ onto itself which leave each frontier point of $S$ fixed. Let $\Theta^{\prime}$ be the class of homeo-
morphisms 5 of the form

$$
x^{\prime}=x, \quad y^{\prime}=\Phi(x, y) \quad \text { or } \quad x^{\prime}=\Phi(x, y), \quad y^{\prime}=y
$$

and which map $S$ onto itself and leave each frontier point of $S$ fixed.

2) The angle $\sin ^{-1}(1 / 4)$ that is needed here lies between 0 and $\pi / 2$.
In the present paragraph we consider homeomorphisms which are defined over certain polygonal arcs contained in $S$ and we shall use these results to show that homeomorphisms of a closed square onto itself can be uniformly approximated to by means of members of $\Xi^{\prime}$, the group generated by $\Theta^{\prime}$.

We require a number of auxiliary lemmas. The following convention is used. If a segment $L$ is such that, apart from its end-points, it belongs to the interior of a closed Jordan domain $T$ then we shall say that $L$ belongs to the interior of $T$. If $X$ is any point set then we denote its interior by $X^{0}$ and its frontier by $\operatorname{Fr} X$.

LEMMA 2. If a simple polygonal are $a q_{0} q_{1} \ldots q_{m} b$, where $m \neq 0$, together with the segment $a b$ bounds a simple closed Jordan domain $T$, then at least one of the segments $a q_{1}, q_{0} q_{2}, \ldots, q_{i-1} q_{i+1}, \ldots, q_{m-1} b$ belongs to the interior of $T$.

Either $q_{0} b$ belongs to $T^{0}$ or there is a vertex $q_{j}, j \neq 0$ such that $a q_{j}$ belongs to $T^{0}$. In the first case we replace $a q_{0} q_{1} \ldots q_{m} b a$ by $q_{0} q_{1} \ldots q_{m} b q_{0}$ and in the second case we replace $a q_{0} q_{1} \ldots q_{m} b a$ by $a q_{0} q_{1} \ldots q_{j-1} q_{j} a$. In either case we obtain a polygonal curve which bounds a domain contained in $T$, has less segments than $a q_{0} q_{1} \ldots q_{m} b a$ and is such that all its segments but one belong to $a q_{0} q_{1} \ldots q_{m} b$. If this new polygonal curve is not a triangle then we repeat the process a finite number of times until we obtain a triangle that bounds a domain contained in $T$ and two of whose sides belong to $a q_{0} q_{1} \ldots q_{m} b$. Now the construction of the new polygon from $a q_{0} q_{1} \ldots q_{m} b$ is such that the only side that does not belong to $a q_{0} q_{1} \ldots q_{m} b$ is contained in $T^{0}$. Thus finally the third side of the triangle which we ultimately obtain is contained in $T^{0}$ and it is also one of the segements $a q_{1}, q_{0} q_{2}, \ldots, q_{i-1} q_{i+1}, \ldots, q_{m-1} b$.

The lemma is proved.

LEMMA 3. Two polygonal arcs $a p_{0} p_{1} \ldots p_{k} b$ and $a q_{0} q_{1} \ldots q_{m} b, m \geqslant 0$, together form the boundary of a simple closed Jordan domain $T$. The arc $a p_{0} p_{1} \ldots p_{k} b$ together with $a b$ bounds a convex set $U$ and those of the vertices $q_{0}, q_{1}, \ldots, q_{m}$ which lie on the same side of $a b$ as does $U$ lie either on the segment ab or interior to $U$, (it is assumed that $U$ has interior points). Also all the points $p_{j}, q_{i}$ lie in the strip bounded by the two lines that are perpendicular to $a b$ and pass through $a$ and $b$ respectively. Then either

(i) the arc $a q_{0} q_{1} \ldots q_{m} b$ together with the segment ab bounds a convex set $\nabla$ that is exterior to $T$, or

(ii) ome of the segments $a q_{1}, q_{0} q_{2}, \ldots, q_{i-1} q_{i+1}, \ldots, q_{m-1} b$ is contained in $T^{0}$.

Let $B$ denote the are $a p_{0} p_{1} \ldots p_{k} b$ and let $K$ denote the convex cover of the are $a q_{0} q_{1} \ldots g_{m} b$. Since all the points $q_{i}$ lie inside the strip bounded Fundamenta Mathematicae. T. XLII. 
by the two lines through $a$ and $b$ perpendicular to $a b$ both $a$ and $b$ are frontier points of $K$. Thus Fr $K$ consists of two arcs that join $a$ to $b$. (The set $K$ will have interior points because the arc $a q_{0} q_{1} \ldots q_{m} b$ has at least three vertices where by a vertex is meant a point which is either an end-point or a point that lies on two segments that lie on distinct lines. We take the notation to imply that the segments $a q_{0}, q_{0} q_{1}$, for example, are not collinear.) Let these two arcs be $A_{1}$ and $A_{2}$. Now no points of $B$ are interior points of $K$ and, of all the vertices of $K$ (which are some of the points $a, b, q_{0}, q_{1}, \ldots, q_{m}$ ), only $a$ and $b$ belong to $B$. Thus $K$ meets $B$ either in the whole segment $a b$ or in just the two points $a$ and $b$. The first case can arise only when $B$ is precisely the segment $a b$. In this case $U$. would have no interior points which is contrary to the hypotheses of the lemma. We suppose then that $B$ meets $K$ in the two points $a$ and $b$.

The two ares $A_{1}$ and $B$ bound a domain say $W_{1}$. Similarly we define $W_{2}$ to be the domain bounded by $A_{2}$ and $B$. Either every segment of $A_{1}$ belongs to $W_{2}^{0}$ or every segment of $A_{2}$ belongs to $W_{1}^{0}$. We choose the notation so that the first of these alternatives holds. If the whole of $A_{1}$ belongs to the arc $a q_{0} q_{1} \ldots q_{m} b$ then it coincides with the whole of this arc and the lemma is proved since (i) is true. If however there is a segment of $A_{1}$ that does not belong to $a q_{0} q_{1} \ldots q_{m} b$ then we can find two points of the set $a, q_{0}, q_{1}, \ldots, q_{m}, b$ say $r_{1}$ and $r_{2}$ such that these points belong to $A_{1}$ and there are no other points of the arc $a q_{0} q_{1} \ldots q_{m} b$ that belong to the segment $r_{1} r_{2}$. The points $r_{1}$ and $r_{2}$ are not necessarily vertices of $K$ but they are vertices of the arc $a q_{0} q_{1} \ldots q_{m} b$.

The points $r_{1}$ and $r_{2}$ are not consecutive vertices of $a q_{0} q_{1} \ldots q_{m} b$ for if they were the whole of the segment $r_{1} r_{2}$ would belong to the arc $a q_{0} q_{1} \ldots q_{m} b$. If they are separated in this sequence by only one other vertex then the segment $r_{1} r_{2}$ itself is of the form required in (ii). If however $r_{1}$ and $r_{2}$ are separated by more than one vertex in the order $a q_{0} q_{1} \ldots q_{m} b$ then the subarc of $a q_{0} q_{1} \ldots q_{m} b$ whose end-points are $r_{1}$ and $r_{2}$, bounds with the segment $r_{1} r_{2}$ a domain contained in $T$. We can apply lemma 2 to it and deduce the existence of a segment as required in (ii).

The lemma is proved.

We now prove a result that will be needed in the next paragraph. Suppose that the square $S$ is cut by a number of segments parallel to the $x$-axis. Let these segments, which join the two sides of $S$ that are parallel to the $y$-axis, be denoted by $A_{1}, A_{2}, \ldots, A_{n}$. We also suppose that the segments $A_{1}$ and $A_{n}$ are sides of $S$ and that the notation is such that the ordinate of the line containing $A_{i}$ is greater than or less than that containing $A_{j}$ according as $i$ is less than or greater than $j$. Denote the totality of these segments by $K$.
THEOREM 2. If $\mathfrak{G}^{-1}$ is a homeomorphism defined over $K \cup$ FrS which leaves every point of the frontier of $S$ fixed and maps each of the segments $A_{i}$ onto a polygonal line contained in $S$, then there is a homeomorphism $\mathfrak{S}$ of the group $\Xi^{\prime}$ such that for any point $p$ of $K \mathfrak{S}\left(\mathfrak{G}^{-1}(p)\right)=p$.

Write $a_{i}, b_{i}$ for the two end points of $A_{i}$ and use the symbols $A_{i}^{\prime}$ for $6^{-1}\left(A_{i}\right)$. By the given conditions $6^{-1}\left(a_{i}\right)=a_{i}$, and $5^{-1}\left(b_{i}\right)=b_{i}$.

We need the following lemmas.

LEMMA 4. If $A_{2}^{\prime}$ is a polygonal line with $t$ segments, $t>1$, then either (a) it bounds with the segment $a_{2} b_{2}$ a convex set that is exterior to the set bounded by $A_{1}^{\prime}, A_{2}^{\prime}$, segment $a_{1} a_{2}$ and segment $b_{1} b_{2}$, or

(b) there is a member $\mathfrak{S}$ of $\Xi^{\prime}$ such that $\mathfrak{H}\left(A_{2}^{\prime}\right)$ is a polygonal line of at most $t-1$ segments and every point of each $A_{j}^{\prime}, j \neq 2$ is a fixed point under $\mathfrak{H}$.

We apply lemma 3 with $a_{2}$ for $a$ and $b_{2}$ for $b$, with $a_{2} a_{1} b_{1} b_{2}$ for $a p_{0} p_{1} \ldots p_{k} b$ and $A_{2}^{\prime}$ for $a q_{0} q_{1} \ldots q_{m} b$. By that lemma if (a) is not true then there are two consecutive segments of $A_{2}^{\prime}$ say $c d$ and $d e$ such that the segment $c e$ is contained in $T^{0}$, where $T$ is the domain bounded by $A_{1}^{\prime}, A_{2}^{\prime}$, $a_{1} a_{2}, b_{1} b_{2}, i . e . c e$ is, apart from its end-points, contained in the interior of $T$. The segment $c e$ does not meet any $A_{j}^{\prime}$ with $j \neq 2$. We next select two points $d^{\prime}$ and $d^{\prime \prime}$ on opposite sides of the line ce such that the closed quadrilateral $c d^{\prime} e d^{\prime \prime}$ is convex, contains $d$ as an interior point, does not meet $A_{j}^{\prime}, j \neq 2$, and meets $A_{2}^{\prime}$ only in the segments $c d$, de. This last requirement can be satisfied because only the end-points of the segment $c e$ belong to $A_{2}^{\prime}$ and thus the triangle cde meets $A_{2}^{\prime}$ only in the segments $c d$ and $d e$. Join $d$ to a point of $c e$ other than $c$ or $e$, by a polygonal line lieing in the interior of $c d^{\prime} e d^{\prime \prime}$, such that each segment of this line is parallel either to the $x$-axis or to the $y$-axis. Let this polygonal line be $d r_{1} r_{2} \ldots r_{s}$, where $r_{s}$ is the point of $c e$.

We shall now show that there is a member of $\Xi^{\prime}$ say $\mathfrak{J}_{1}$ such that

$$
\mathfrak{I}_{1}(d)=r_{1} ; \quad \Im_{1}(c d)=c r_{1} ; \quad \Im_{1}(d e)=r_{1} e,
$$

and such that every point on the frontier of or exterior to the quadrilateral $c d^{\prime} e d^{\prime \prime}$ is fixed under $\widetilde{\Im}_{1}$.

Suppose for the moment that such a homeomorphism exists, then the lemma follows. For just as we have defined $\mathfrak{J}_{1}$ so we can define $\mathfrak{J}_{i}$ such that

$$
\mathfrak{I}_{i}\left(r_{i-1}\right)=r_{i}, \quad \mathfrak{I}_{i}\left(c r_{i-1}\right)=c r_{i}, \quad \Im_{i}\left(r_{i-1} e\right)=r_{i} e, \quad i=2, \ldots, s
$$

and every point on the frontier of or exterior to the quadrilateral $c d^{\prime} e d^{\prime \prime}$ is fixed under $\mathfrak{I}_{i}$. Then the homeomorphism $\mathfrak{I}_{s} \mathfrak{J}_{s-1} \ldots \mathfrak{I}_{1}$ maps $c d$ onto $c r_{s}$ and de onto $r_{s} e$. Since all the other points of $A_{2}^{\prime}$ are fixed it reduces the 
number of segments of $A_{2}^{\prime}$ by at least one. Also every point of every $A_{j}^{\prime}$, $j \neq 2$ is fixed and the lemma is established.

Thus we have only to construct the homeomorphism $\mathfrak{I}_{1}$. Suppose that $d r_{1}$ is parallel to the $y$-axis. In the other case we use the same argument with $x$ and $y$ interchanged. On a particular line $x=x^{\prime}, \Im_{1}$ is defined as follows. All points exterior to or on the frontier of $c d^{\prime} e d^{\prime \prime}$ are fixed. If the line $x=x^{\prime}$ meets the pair of segments $c d, d e$ in one point say $p$, then it also meets the pair of segments $c r_{1}, r_{1} e$ in one point $q$. Define $\mathfrak{J}_{1}(p)$ to be $q$ and complete the definition of $\mathfrak{J}_{1}$ on $x=x^{\prime}$ by linearity. If the line $x=x^{\prime}$ meets $c d$, de in two points say $p^{\prime}$ and $p^{\prime \prime}$ and the ordinate of $p^{\prime}$ is greater than that of $p^{\prime \prime}$, then the line also meets the segments $c r_{1}, r_{1} e$ in two points which we may call $q^{6}$ and $q^{\prime \prime}$ where the ordinate of $q^{\prime}$ is greater than that of $q^{\prime \prime}$. Define $\mathfrak{I}_{1}\left(p^{\prime}\right)$ to be $q^{\prime}$ and $\mathfrak{I}_{1}\left(p^{\prime \prime}\right)$ to be $q^{\prime \prime}$ and complete the definition of $\mathfrak{I}_{1}$ on the line by linearity. If the line $x=x^{\prime}$ contains the whole of the segment $c d$ or $d e$ then it also contains the point $r_{1}$. Define $\mathfrak{I}_{1}(d)$ to be $r_{1}$ and complete as before by linearity. If the abscissae of the two points $d^{\prime}$ and $d^{\prime \prime}$ are equal to one of or lie between the abscissae of $c$ and $e$ then the definition of $\widetilde{I}_{1}$ is complete. Otherwise we still have to define it for those lines $x=x^{\prime}$ that meet the quadrilateral $c d^{\prime} e d^{\prime \prime}$ but do not meet the pair of segments $c d, d e$. In this case there is a line $x=x^{\prime \prime}$ on which $\Im_{1}$ has been defined, and which passes through one or more of the points $c, d, e$, and which is such that the segment of the line $x=x^{\prime \prime}$ that is interior to the quadrilateral $c d^{\prime} e d^{\prime \prime}$, say $t u$, forms with either $d^{\prime}$ or $\vec{d}^{\prime \prime}$, say $\vec{d}^{\prime \prime}$, a triangle that contains that part of the line $x=x^{\prime}$ that is interior to the quadrilateral $c d^{\prime} e d^{\prime \prime}$. Let the part of $x=x^{\prime}$ that is contained in $c d^{\prime} e \vec{a}^{\prime \prime}$ be the segment $v w$. To define $\Im_{1}$ on $v w$, join $d^{\prime \prime}$ to a point say $z$ on $v w$, produce to meet $t u$ in $z^{\prime}$. Let $\widetilde{I}_{1}\left(z^{\prime}\right)$, which has been defined to be a point of $t u$, be the point $z_{1}^{\prime}$. Join $z_{1}^{\prime}$ to $d^{\prime \prime}$ cutting $v w$ in $z_{1}$. Define $\mathfrak{I}_{1}(z)$ to be $z_{1}$.

Then $\mathfrak{J}_{1}$ has been completely defined, it is of the form

$$
(x, y) \rightarrow\left(x^{\prime}, y^{\prime}\right) \quad \text { where } \quad x^{\prime}=x, \quad y^{\prime}=\Phi(x, y),
$$

and has the properties stated in (16).

The proof of the lemma is complete.

LEMMA 5. A rectangle $T$ is given with its sides parallel to the coomdinate axes. A homeomorphism $\Omega$ defined over the frontier of $T$ is of the form.

$$
(x, y) \rightarrow\left(x^{\prime}, y\right) \quad \text { where } \quad x^{\prime}=\Phi(x, y) .
$$

Further $\mathfrak{\Omega}$ leaves fixed the points of the sides of $T$ that are parallel to the $y$-axis. Then there is a homeomorphism which is of the form (17), maps the whole of $T$ onto itselt and coincides with $\Re$ on $\operatorname{Fr} T$.
Suppose that $T$ is the rectangle $a_{1} \leqslant x \leqslant a_{2} ; b_{1} \leqslant y \leqslant b_{2}$. Consider the two rectangles

$$
\begin{array}{lll}
T_{1}: & a_{1} \leqslant x \leqslant a_{2}, & \frac{1}{2}\left(b_{1}+b_{2}\right) \leqslant y \leqslant b_{2}, \\
T_{2}: & a_{1} \leqslant x \leqslant a_{2}, & b_{1} \leqslant y \leqslant \frac{1}{2}\left(b_{1}+b_{2}\right) .
\end{array}
$$

In $T_{1}$ define the homeomorphism $\mathfrak{H}$ by $(x, y) \rightarrow\left(x^{\prime}, y\right)$ where

$$
x^{\prime}=x+\left(\Phi\left(x, b_{2}\right)-x\right)\left(y-\frac{1}{2}\left(b_{1}+b_{2}\right)\right) / \frac{1}{2}\left(b_{2}-b_{1}\right) .
$$

In $T_{2}$ define $\mathfrak{H}$ by $(x, y) \rightarrow\left(x^{\prime}, y\right)$ where

$$
x^{\prime}=x+\left(\Phi\left(x, b_{1}\right)-x\right)\left(\frac{1}{2}\left(b_{1}+b_{2}\right)-y\right) / \frac{1}{2}\left(b_{2}-b_{1}\right) .
$$

It may be verified that $\mathfrak{S}$ is a homeomorphism with the properties stated in the lemma.

Levras 6. There is a member $\mathfrak{H}$ of $\Xi^{\prime}$ such that $\mathfrak{S}\left(A_{j}^{\prime}\right)=A_{j}, j=1, \ldots, n$.

Consider $A_{2}^{\prime}$. By lemma 4 , either $A_{2}^{\prime}$ is an are convex with respect to $a_{2} b_{2}$ and $A_{2}^{\prime}$ lies on the same side of $a_{2} b_{2}$ as $A_{1}^{\prime}$, or we can find $\mathfrak{I}_{1}$ of $\Xi^{\prime}$ which reduces the number of segments of $A_{2}^{\prime}$ and leaves every point of every $A_{j}^{\prime}, j \neq 2$, fixed. By successive repetitions of this argument it follows that we can find a member of $\Xi^{\prime}$ say $\widetilde{F}_{1}$ such that either $\widetilde{F}_{1}\left(A_{2}^{\prime}\right)$ is the straight line segment $a_{2} b_{2}$ or it is convex with respect to $a_{2} b_{2}$ and lies on the same side of $a_{2} b_{2}$ as does $A_{1}^{\prime}$. In either case $\widetilde{F}_{1}\left(A_{2}^{\prime}\right)$ together with the segments $a_{2} a_{3}, a_{3} b_{3}, b_{3} b_{2}$, bounds a conrex set. We may now apply lemma 3 to $A_{3}^{\prime}$ exactly as we applied it to $A_{2}^{\prime}$ in the proof of lemma 4 . We obtain $\widetilde{\mho}_{2}$ of $\Xi^{\prime}$ such that every point of every $A_{j}^{\prime}, j>3$, and of $A_{1}$, $\widetilde{\mho}_{1}\left(A_{2}^{\prime}\right)$ is fixed, and such that $\widetilde{\mho}_{2}\left(A_{3}^{\prime}\right)$ is convex with respect to the segment $a_{3} b_{3}$ and lies on the same side of $a_{3} b_{3}$ as does $A_{1}^{\prime}$, or alternatively $\widetilde{\mho}_{2}\left(A_{3}^{\prime}\right)$ is a straight line segment. Proceeding in this fashion we eventually arrive at a member $\widetilde{F}$ of $\Xi^{\prime}$ such that each $\mathbb{F}\left(\mathcal{A}_{j}^{\prime}\right)$ is either the segment $a_{j} b_{j}$ or bounds with $a_{j} b_{j}$ a convex set.

Now every line parallel to the $y$-axis that meets $\widetilde{F}\left(A_{j}^{\prime}\right)$ at all does so in exactly one point and also meets $A_{j}$ in exactly one point. Suppose that such a line meets $\widetilde{\mho}\left(A_{j}^{\prime}\right)$ in $x_{j}$ and $A_{j}$ in $y_{j}$. Then the points $x_{1} x_{2} \ldots x_{n}$ oceur on this line in the same order as $y_{1} y_{2} \ldots y_{n}$. We define a homeomorphism on this line by mapping $x_{j}$ onto $y_{j}$ and making the mapping linear between $x_{j}$ and $x_{j+1}$. Such a mapping is defined for all the points of $S$. For points outside $S$ we define each point to be its own image. Denote this mapping by $\mathfrak{L}$. Then $\mathfrak{L} \widetilde{J}$ is a member of $\Xi^{\prime}$ with the property stated in the enunciation of the lemma. 
We can now complete the proof of theorem 2. This theorem is nearly contained in lemma 6 but although that lemma provides a homeomorphism that maps $A_{j}^{\prime}$ onto $A_{j}$ we do not know that it coincides with (5) for each point $p$ of $\left(^{-1}(p)\right.$. To secure this result we proceed as follows.

The homeomorphism $\mathfrak{L} \mathfrak{F}_{\mathfrak{H}^{-1}}$ maps each segment $A_{\boldsymbol{J}}$ onto itself and leaves each point of the frontier of $S$ fixed. If lemma 5 is applied to the rectangle that is bounded by $A_{j}, A_{j+1}$, and the two segments $a_{j} a_{j+1}$, $b_{j} b_{j+1}$ then there is a homeomorphism of the form (17) say $\mathfrak{M}_{j}$ that maps this rectangle onto itself and coincides with $\mathfrak{L} \mathfrak{F}^{-1}$ on the frontier of this rectangle. Denote by $\mathfrak{M}$ the homeomorphism of the whole plane onto itself that coincides with $\mathfrak{M}_{j}$ in the rectangle in which it is defined and leaves every point exterior to $S$ fixed. Then for any point $p$ of $K \mathfrak{M}(p)$ is the point $\mathfrak{L} \mathfrak{F} \mathfrak{F}^{-1}(p)$. Thus $p=\mathfrak{M}^{-1} \mathfrak{R F} \mathfrak{F}^{-1}(p)$, that is to say the homeomorphism $\mathfrak{M}^{-1} \mathfrak{S} \mathfrak{F}$ which is a member of the group $\Xi^{\prime}$ has the property required in the theorem.

\section{\$ 3. Homeomorphisms of a closed square onto itself}

As before let $S$ denote a closed square with sides parallel to the axes. In this paragraph all the homeomorphisms concerned map $S$ onto itself and leave the frontier points of $S$ fixed. This class of homeomorphisms is denoted by $\Gamma$. Again we write $\Theta^{\prime}$ for the class of homeomorphisms that belong both to $\Gamma$ and to $\Theta$, and we write $\Xi^{\prime}$ for the group generated by finite superpositions of members of $\Theta^{\prime}$.

THEOREM 3. If $\mathfrak{G}$ is a homeomorphism belonging to the class $\Gamma$, then, given a positive number $\varepsilon$ there exists a homeomorphism $\mathfrak{H}$ of $\boldsymbol{\Xi}^{\prime}$ such that

$$
\delta^{\prime}(\mathfrak{S},(\mathfrak{5})=\underset{p \in \mathcal{S}}{\operatorname{up} . \operatorname{bd}} \varrho(\mathfrak{S}(p), \mathfrak{G}(p))<\varepsilon .
$$
that

Let $l$ be the side length of $S$ and choose a positive integer $n$ so large

$$
4 \cdot 2^{1 / 2} l<n \varepsilon .
$$

Divide $S$ into $n^{2}$ equal squares each of side length $l / n$ and let the vertices of these squares be $a_{i j}, i=1,2, \ldots, n+1, j=1,2, \ldots, n+1$, where the vertices of $S$ itself are $a_{11}, a_{1, n+1}, a_{n+1, n+1}, a_{n+1,1}$. Denote the segment $a_{i 1} a_{i, n+1}$ by $A_{i}$ and the segment $a_{1 j} a_{n+1, j}$ by $B_{j}$. Also write $\mathfrak{G}^{-1}\left(A_{i}\right)=A_{i}^{\prime}$, $\mathfrak{G}^{-1}\left(a_{i j}\right)=a_{i j}^{\prime}, \mathfrak{G}^{-1}\left(B_{j}\right)=B_{j}^{\prime}$; and let $E_{i j}$ be the subarcs of $A_{i}^{\prime}$ with end points $a_{i j}^{\prime}, a_{i, j+1}^{\prime}$ and $F_{i j}$ be the subarcs of $B_{j}^{\prime}$ with end points $a_{i j}^{\prime}, a_{i+1, j}^{\prime}$. Let the axes be such that the $x$-axis is parallel to each $A_{i}$ and the $y$-axis is parallel to each $B_{j}$.

Define $\eta$ to be a positive number, less than the least distance apart of any pair of nonintersecting arcs $E_{i j}$ or $F_{i j}$.
Let $C_{i j}$ be the closed circle whose centre is $a_{i j}^{\prime}$ and whose radius is $\eta / 3$. No two of the circles $C_{i j}$ intersect; let $C$ denote their point-set union. The arc $A_{j}^{\prime}$ contains a subarc that is minimal with respect to the property of joining $C_{j i}$ to $C_{j, i+1}$, and which we denote by $L_{j i}, j=1,2, \ldots, n$, $i=1,2, \ldots, n+1$. Similarly there is a minimal subarc of $B_{i}^{\prime}$ that joins $C_{j i}$ to $C_{j+1, i}$, say $M_{j i}, j=1,2, \ldots, n, i=1,2, \ldots, n+1$.

Any two segments of the form $A_{j}$ or $B_{i}$ either do not meet or do so in one of the points $a_{j i}$. Thus any two of the arcs $A_{j}^{\prime}, B_{i}^{\prime}$ either do not meet at all or if they do meet their point of intersection belongs to the set $C^{0}$. Thus all the arcs of the form $L_{i j}$ and $M_{k r}$ are disjoint from one another. Also of all the circles $C_{i j}$ the are $L_{p q}$ meets only two namely the two circles $C_{p q}$ and $C_{p, q+1}$, similarly $M_{r s}$ meets only the two circles $C_{r s}$ and $C_{r+1, s}$. For suppose that the arc $\dot{L}_{p q}$ met a circle other than the two stated above, say it met $C_{t u}$, then on the one hand $L_{p q}$ is a subset of $E_{p q}$ and is thus at a distance of at least $\eta$ from any of the $\operatorname{arcs} E_{i j}$ or $F_{i j}$ which do not actually meet $E_{p q}$ and on the other hand $\alpha_{t u}^{\prime}$ lies on two ares of the form $E_{i j}$ and on two arcs of the form $F_{i j}$. It follows that $a_{t u}^{\prime}$ lies on an are of one of these two types that does not meet $E_{p q}$. Thus the point $a_{t u}^{\prime}$ is distant at least $\eta$ from $L_{p q}$ and $L_{p q}$ does not meet $C_{t u}$. In fact $L_{p q}$ is distant at least $2 \eta / 3$ from $C_{t u}$.

Let $\eta_{1}$ be a positive number less than the distance apart of any two $\operatorname{arcs} L_{i j}$ or $M_{r s}$ and let $\eta_{0}$ be the smaller of the two numbers $\eta$ and $\eta_{1}$. Let $P_{i j}$ be a polygonal line joining the end-points of $L_{i j}$ and lieing both inside $S$ and within a distance of $\eta_{0} / 3$ of $L_{i j}$ and outside $C_{i j}^{0}$ and $C_{i, j+1}^{0}$, $i=2,3, \ldots, n, j=1,2, \ldots, n$. Let the point of intersection of the arc $L_{i j}$ with the circle $C_{i j}$ be $l_{i j}^{\prime}$ nad the point of intersection of $L_{i j}$ with $C_{t, j+1}$ be $l_{i j}^{i}$. Let $P_{i j}^{\prime}$ be the polygonal line consisting of segment $a_{i j}^{\prime}{ }_{i j}^{\prime}$, polygonal line $P_{i j}$, and segment $l_{i j}^{\prime \prime} a_{i, j+1}^{\prime}$. We also suppose that $F_{1 j}^{\prime}$ is the segment $a_{1 j} c_{1, j+1}$ and that $P_{n+1, j}^{\prime}$ is the segment $a_{n+1, j} a_{n+1, j+1}$. Finally let $P_{i}$ denote the union $\bigcup_{j=1}^{n} P_{i j}^{\prime}, i=1,2, \ldots, n+1$.

Next define polygonal lines $Q_{j}$ in the same way as $P_{j}$ has been defined but using the minimal arcs $M_{r s}$ in place of the minimal ares $\left.L_{i j}{ }^{3}\right)$.

The polygonal lines $P_{i}, Q_{j}$ approximate to $A_{i}^{\prime}$ and $B_{j}^{\prime}$ respectively and have the following properties:

(i) $P_{i}$ intersects $Q_{j}$ in exactly one point namely $a_{i j}^{\prime}$.

(ii) Of the points $a_{i j}^{\prime} ; P_{i}$ contains in order the points $a_{i 1}^{\prime}, a_{i 2}^{\prime}, \ldots, a_{i, n+1}^{\prime}$.

(iii) Of the points $a_{i j}^{\prime} ; Q_{j}$ contains in order the points $a_{1 j}^{\prime}, a_{2 j}^{\prime}, \ldots, a_{n+1, j}^{\prime}$.

(iv) The four sides of the square $S$ are $P_{1}, P_{n+1}, Q_{1}, Q_{n+1}$.

s) $Q_{r s}, Q_{r s}^{\prime}$ are defined first with respect to $M_{t s}$. 
Next let $\Omega$ be any homeomorphism of the class $\Gamma$ such that

$$
\Re\left(P_{j}\right)=A_{j}, \quad \mathfrak{K}\left(Q_{j}\right)=B_{j} .
$$

It is clear that such a homeomorphism exists. If $z$ is a point of $S$ then it belongs to a domain bounded by four ares of which two are of the form $E_{i j}$ and two are of the form $F_{r s}$. $\approx$ may belong to more than one such domain. (We take these domains to be closed.) Suppose that $z$ belongs to the domain bounded by $E_{i j}, F_{i, j+1}, E_{i+1, j}, F_{i j}$. If $2 \leqslant i, j \leqslant n-1$, then $z$ also belongs to the domain bounded by the polygonal lines $P_{i-1, j-1}^{\prime}, P_{i-1, j}^{\prime}, P_{i-1, j+1}^{\prime}, Q_{i-1, j+1}^{\prime}, Q_{i, j+1}^{\prime}, Q_{i+1, j+1}^{\prime}, P_{i+1, j+1}^{\prime}, P_{i+1, j}^{\prime}, P_{i+1, j-1}^{\prime}, Q_{i+1, j-1}^{\prime}$, $Q_{i, j-1}^{\prime}, Q_{i-1, j-1}^{\prime}$. A similar statement is true when one or both of $i$ or $j$ is one of 1 or $n$. Thus we have

$$
\varrho\left(\Re(z),(\mathfrak{G}(z)) \leqslant 2 \cdot \dot{2}^{1 / 2}(l / n)<\frac{1}{2} \varepsilon .\right.
$$

Hence it is sufficient to show that there is á member $\mathfrak{H}$ of $\Xi^{\prime}$ such that for every point $z$ of $S$

$$
\varrho\left((\mathfrak{N}(z), \mathfrak{J}(z)) \leqslant \frac{1}{2} \varepsilon\right.
$$

By theorem 2 there is a member $\mathfrak{I}$ of $\Xi^{\prime}$ such that for any point $p$ of any of the polygonal lines $P_{j} j=1,2, \ldots, n+1, \mathfrak{I}(p)=\mathfrak{K}(p)$. Consider the homeomorphism $\Omega \mathfrak{I}^{-1}$. This mapping leaves fixed each point of the frontier of $S$ and of each of the segments $A_{j}$. Denote by $T_{j}$ that rectangle which has $A_{j}$ and $A_{j+1}$ as two opposite sides. $T_{j}$ is contained in $S$ and is mapped onto itself by $\mathcal{H}^{-1}$. Thus for any point $p$ of $S$ the ordinate of $\mathfrak{N}(p)$ differs from that of $\mathfrak{I}(p)$ by at most the width of one of the rectangles $T_{j}, i$. e. by at most $l / n$.

Now the homeomorphism $\mathfrak{A}^{-1}$ not only maps $T_{j}$ onto itself but also leaves each point of the frontier of $T_{j}$ fixed, and (even though $T_{j}$ is a rectangle and not a square) all the preceding argument is valid with $T$ in place of $S$. Thus by an argument similar to that used in theorem 2 with $x$ and $y$ interchanged we can show that there is a homeomorphism $\mathfrak{F}_{j}$ which belongs to $\Xi$, maps each point of the frontier of $T_{J}$ onto itself and is such that for any point $p$ of $T_{J}$ the abscissae of $\widetilde{F}_{j}(p)$ and $\Re_{\mathfrak{S}}^{-1}(p)$ differ by at most $l / n$.

Define $\mathfrak{F}$ to be the homeomorphism which coincides with $\mathfrak{F}_{j}$ in $T_{j}$ $j=1,2, \ldots, n+1$, and leaves every point of $E$ exterior to $S$ fixed. Then $\mathfrak{F}$ belongs to $\Xi^{\prime}$ and for any point $p$ of $S$ we have:

(i) The ordinates of $\mathfrak{F}(p)$ and $\Omega^{-1}(p)$ differ by at most $l / n$ because these points both belong to the same rectangle $T$.

(ii) The abscissae of $\mathfrak{F}(p)$ and $\mathcal{R}^{-1}(p)$ differ by at most $l / n$.
Hence

$$
\varrho\left(\left(\mathfrak{F}(p), \mathfrak{H}^{-1}(p)\right) \leqslant 2^{1 / 2}(l ! n)<-\frac{1}{2} \varepsilon\right.
$$

Now for any point $q$ of $S$ there is a point $p$ such that $p=\mathfrak{J}(q)$, and thus for any point $q$ of $S$

$$
\varrho(\mathfrak{F} \mathfrak{J}(q), \mathfrak{R}(q))=\varrho\left(\widetilde{\mathfrak{F}}(p), \mathfrak{R}^{-1}(p)\right)<\frac{1}{2} \varepsilon
$$

and this is the inequality (19) as required with $\mathfrak{F} \mathfrak{J}$ as the homeomorphism $\mathfrak{5}$.

Theorem 3 is proved.

\section{§ 4. Non-nniform approximations}

In this paragraph we use theorem 3 to establish the result stated in the introduction.

THEOREM 4. If 5 is any given homeomorphism of the plane onto itself, then there exists a sequence of members of $\Xi$ say $\left\{\mathfrak{S}_{n}\right\}$ such that for every point $q$ of the plane, $\mathfrak{S}_{n}(q)$ tends to $\mathfrak{G}(q)$ as $n$ tends to infinity.

Let $C=C(p, R)$ denote the set of points of the plane whose distance from the fixed point $p$ is not more than $R$, and let $\mathfrak{G}(C)$ be $D$. It is sufficient to show that given two positive numbers $R$ and $\varepsilon$, there is a member of $\Xi$ say $\mathfrak{H}$, such that the distance apart of the points $\mathfrak{H}(z)$ and $\mathfrak{G}(z)$ for all points $z$ of $C(p, R)$ is less than $\varepsilon$.

Let $S$ be a square so large that it contains both $C$ and $D$ in its interior. We show first that there is a homeomorphism $\mathfrak{I}$ of $E$ onto itself that coincides with $\mathfrak{G}$ on $C$ and leares each point of the frontier of $S$ fixed. Let $S_{1}$ be the set $S^{0}-C$ and let $S_{2}$ be the set $S^{0}-D$. Both $S_{1}$ and $S_{2}$ are open, connected, and doubly connected sets. Thus there are conformal mappings say $\mathfrak{M}_{1}$ and $\mathfrak{M}_{2}$ such that $\mathfrak{M}_{1}\left(S_{1}\right)$ is an annulus $A_{1}$ and $\mathfrak{M}_{2}\left(S_{2}\right)$ is an annulus $\mathcal{A}_{2}$. Since $\operatorname{Fr} S, \operatorname{Fr} C$, and FrD are all Jordan curves we can extend $\mathfrak{M}_{1}$ and $\mathfrak{M}_{2}$ to be homeomorphic over the closures of $S_{1}$ and $S_{2}$. We use the same notation $\mathfrak{M}_{1}$ and $\mathfrak{M}_{2}$ for these two homeomorphisms of the closed sets. Let $\mathfrak{C}$ be a homeomorphism of the closure of $A_{2}$ onto the closure of $A_{1}$ such that for any point $p$ of $\operatorname{Fr} S \quad \mathfrak{C M}_{2}(p)=\mathfrak{M}_{1}(p)$. Let the circles bounding $A_{1}$ be $K$ and $L$ and suppose that $L$ is the image under $\mathfrak{M}_{1}$ of $\operatorname{Fr} S$.

We next define a homeomorphism of $K$ onto itself say $\mathcal{K}$, as follows. For $q$ belonging to $K$ write

$$
\mathfrak{R}(q)=\mathfrak{C M}_{2}\left(\mathfrak{S} \mathfrak{M}_{1}^{-1}(q)\right.
$$


Now we can extend $\Re$ so that we obtain a homeomorphism of the closure of $A_{1}$ onto itself say $\mathfrak{J}$ with the properties,

$$
\text { if } p \in K \quad \mathfrak{I}(p)=\mathfrak{N}(p), \quad \text { if } \quad p \in L \quad \mathfrak{I}(p)=p .
$$

Now define $\mathfrak{J}$ as follows. For $p \in C, \mathfrak{I}(p)=\mathfrak{b}(p)$; for $p \in \operatorname{Fr} S \mathfrak{S}(p)=p$; for $p \epsilon S^{0}-C \mathfrak{I}(p)=\mathfrak{M}_{2}^{-1} \mathfrak{C}^{-1} \mathfrak{J}_{\mathfrak{M}_{1}}(p)$; for $p \notin S \mathfrak{S}(p)=p$. Then $\mathfrak{J}$ is a homeomorphism of the required form.

By $\S 3$ we can find a member $\mathfrak{S}$ of $\Xi$ such that for every point $z$ of $S, \mathfrak{S}$ and $\mathfrak{I}$ differ by at most $\varepsilon$. The homeomorphism $\mathfrak{H}$ has the required property and the theorem 4 is proved.

Reşu par la Rédaction le 5. 4. 1954

\section{Über eine Abschwächung des Auswahlpostulates}

von

\section{W. Kinna (Solingen) und K. Wagner (Köln)}

Es sei $M$ eine Menge. Wir bezeichnen die Elemente von $M$ mit $a, b, \ldots$, hingegen die Teilmengen von $M$ mit $A, B, \ldots$ Die Potenzmenge von $M$ (d. h. die Menge sämtlicher Teilmengen von $M$, einschließlich der leeren Menge 0 ) bezeichnen wir mit $M^{*}$.

Definition. Wir sagen, eine Menge $M$ habe die Eigenschaft (E), wenn es eine eindeutige Abbildung $\varphi$ von $M^{*}$ in sich gibt, so daß für jedes aus mindestens zwei Elementen bestehende $A \subseteq M$ gilt:

$$
\left.0 \subset \varphi(A) \subset A^{1}\right) \text {. }
$$

Unsere Bedingung (E) steht in einem engen Zusammenhang mit dem bekannten ${ }^{2}$ ) Auswahlpostulat. Wie man ohne weiteres sieht, ist (E) formal schwächer als das Auswahlpostulat. Man kann aber außerdem leicht zeigen, daß das Kontinuum $C$ noch (E) erfüllt. Denn mittels der unendlichen Folge der rationalen Zahlen gelingt es, da diese relativ zu $C$ dicht liegen, jedes aus mindestens zwei Zahlen bestehende $A \subseteq C$ in ein echtes Anfangsstück und ein echtes Enđstück von $A$ zu zerlegen. Versteht man dann unter $\varphi(A)$ dieses echte, nicht leere Anfangsstück von $A$, so folgt unmittelbar unsere Behauptung. Wir sehen, unșer (E) ist nichts weiter als eine gewisse Abschwächung des Auswahlpostulates.

SaTz 1. Jede Menge $M$ mit der Eigenschaft (E) läßt sich ordnen.

Beweis. Wir setzen $\left.{ }^{3}\right) \bar{\varphi}(A)=A-\varphi(A)$ für jedes $A \subseteq \underline{\subseteq} M$. Dann folgt auch

$$
0 \subset \bar{\varphi}(A) \subset A
$$

für jedes aus mindestens zwei Elementen bestehende $A \subseteq \underline{C} M$. Ferner folgt

1) D. h. also, $\varphi(A)$ ist eine echte, nicht leere Teilmenge von $A$. Die vorliegende A rbeit enthält die wesentlichen Ergebnisse der (unveröffentlichten) Dissertation (Köln 1952) des erstgenannten Verfassers, der durch diese vom letztgenannten Verfasser stammende Definition sowie durch den Satz 1 angeregt wurde.

2) Siehe [7], Abschnitt 2, S. 514 .

s) Es ist im folgenden bequem, für die aus nur einem Element bestehenden $A$, also (kurz geschrieben) für die $a \in M$, einfach $\varphi(a)=a$ vorauszusetzen. Ferner setzen wir im folgenden für die Nullmenge $\varphi(0)=0$ voraus. 